\title{
NOUVELLE
}

\section{Les péricytes des îlots de Langerhans régulent localement le diamètre des capillaires et le flux sanguin}

Laure Alexandre-Heymann, Étienne Larger
Institut Cochin, Inserm U1016, Hôpital Cochin - APHP, service de diabétologie, 123, boulevard de Port-Royal, 75014 Paris, France.

laure.alexandre@inserm.fr etienne.larger@inserm.fr
> Le diabète de type 2 (DT2) est un ensemble de maladies caractérisées par la combinaison d'anomalies de la sensibilité et de la sécrétion d'insuline, dont les déterminants principaux sont le surpoids, la sédentarité et l'âge. Cependant, si on a beaucoup entendu parler d'épidémie de diabète de type 2 associée à l'augmentation de la prévalence de l'obésité, on a ignoré l'augmentation de prévalence du diabète de type 2 associée au vieillissement de la population. Pourtant, une grande étude écossaise a bien souligné que l'âge au diagnostic avançant, le surpoids a un rôle moindre dans le déterminisme du diabète [1].

Cette observation pourrait être en partie expliquée par la dysfonction des vaisseaux pancréatiques, dont le rôle dans les anomalies de sécrétion de l'insuline du diabète de type 2 est évoqué depuis une dizaine d'années $[2,3](\rightarrow)$.

$\rightarrow$ Voir la Synthèse de $\varepsilon$. Larger, $m / s$ n० 8-9, août-sepCependant, jusqu'à tembre 2003, page 840

présent, seul un nombre restreint d'études expérimentales supporte cette hypothèse. C'est l'objet du travail publié en mars 2018 par Almaça et ses collaborateurs, qui souligne un rôle potentiellement important des capillaires insulaires dans la physiopathologie du diabète, en montrant comment les flux sanguins sont étroitement associés à la sécrétion d'insuline [4]. Les péricytes $^{l}(P C)$ jouent un rôle important dans la fonction des capillaires,

${ }^{1}$ Les péricytes sont de cellules mésenchymateuses non-différenciées. Ces cellules contractiles sont localisées au niveau de la lame basale des endothéliums. la régulation de la perméabilité vasculaire et le flux sanguin [5]. Ainsi, les PC régulent le diamètre des capillaires de la rétine, du cerveau et du rein $[6,7]$, et il a été montré que la perte des péricytes est le premier signe anatomique de la rétinopathie diabétique. Un premier travail avait montré que la destruction des péricytes par un système impliquant le facteur de transcription Nkx3.2 sous l'effet de la toxine diphtérique conduisait à des anomalies de sécrétion de l'insuline avec hyperglycémie et dédifférenciation des cellules bêta [8]. Almaça et ses collaborateurs ont donc cherché à préciser le rôle des péricytes des îlots de Langerhans et leur association à la sécrétion d'insuline.

\section{Les péricytes recouvrent en grande partie les capillaires insulaires}

La première partie de leur travail montre que chez la souris comme chez l'homme, les PC représentent environ $3 \%$ des cellules de l'îlot de Langerhans, et sont pourvus d'extensions cytoplasmiques recouvrant une grande partie des cellules endothéliales: la surface des capillaires recouverte par les $P C$ est estimée à environ $40 \%$. Chez les quelques sujets ayant un diabète de type 2 qui ont pu être étudiés dans le cadre de ce travail, le nombre de péricytes par îlot était plus faible que chez les témoins, et cette réduction semblait associée à la durée de diabète.

\section{Les péricytes insulaires ont des} propriétés contractiles

Cinquante pour cent des $P C$ insulaires expriment l'isoforme d'actine présent dans les cellules musculaires lisses ( $\alpha S M A$, smooth muscle actin). Les coupes de pancréas ex vivo (CPEV) représentent une nouvelle technologie offrant la possibilité d'étudier in vitro les régulations de fonction des îlots de Langerhans dans leur environnement naturel [9]. Les auteurs ont ainsi pu étudier les mouvements calciques intracellulaires au sein des PC en réponse à divers stimulus. Le modèle a été validé en montrant une vasoconstriction des capillaires insulaires en réponse à l'endothéline 1 : on observe alors une augmentation de la concentration du calcium cytosolique dans les PC, suivie d'une contraction intense et prolongée d'une partie des capillaires insulaires.

Plusieurs types de vasoconstricteurs ont été testés, mais c'est toujours le même sous-groupe de capillaires insulaires qui répond. Ce sous-groupe représente environ $20 \%$ des capillaires de l'îlot, ce qui correspond à la proportion de capillaires recouvert par des PC exprimant l' $\alpha$ SMA.

L'activation des cellules bêta induit vasodilatation et augmentation du flux sanguin via l'inhibition de l'activité des péricytes

Après avoir montré que les $P C$ sont présents autour des capillaires insulaires et peuvent en contrôler les modifications de diamètre en réponse à différents stimulus, les auteurs ont cherché à comprendre les effets des variations glycémiques sur ce système complexe.

Une augmentation de la concentration de glucose appliquée aux CPEV conduit à une diminution de la concentration en calcium cytosolique dans les 


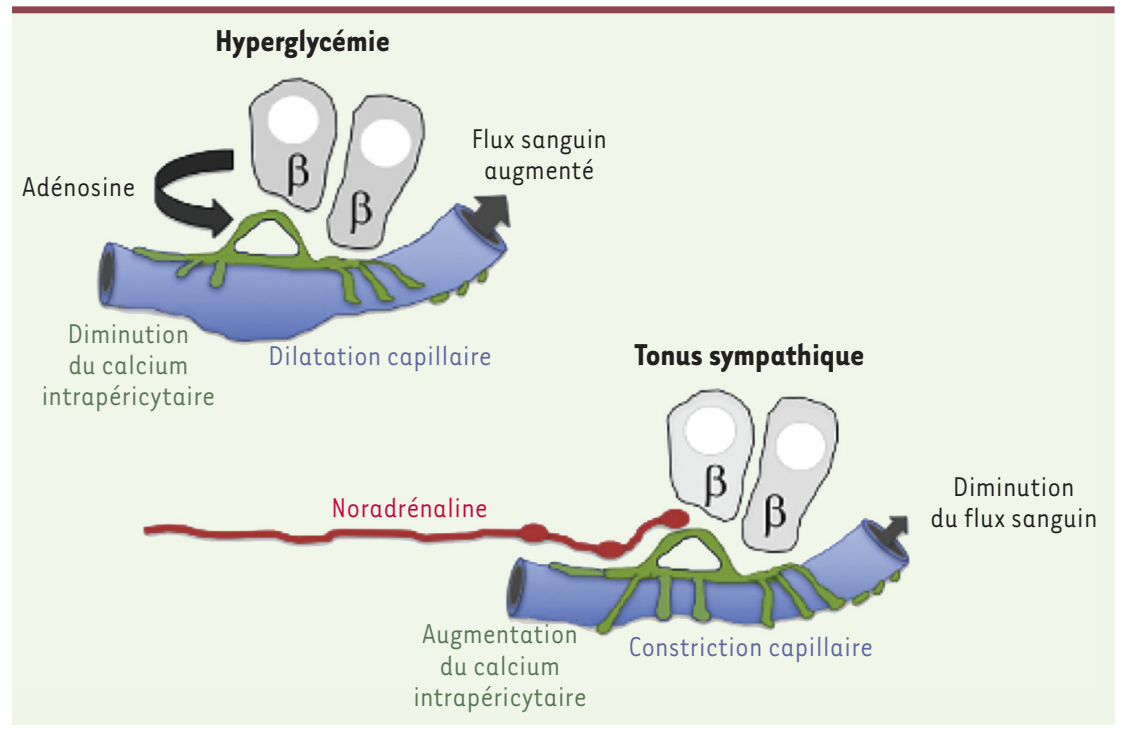

Figure 1. Régulation du flux sanguin insulaire. Les péricytes (en vert) représentent environ $3 \%$ des cellules des îlots de Langerhans et sont pourvus d'extensions cytoplasmiques recouvrant une grande partie des cellules endothéliales. L'augmentation de l'activité des cellules bêta-pancréatiques est associée à une augmentation de la concentration d'adénosine extracellulaire qui reproduit les effets de l'augmentation du taux de glucose sur les péricytes insulaires. L'adénosine induit une diminution $\mathrm{du} \mathrm{Ca}^{2+}$ intrapéricytaire, une dilatation du vaisseau et une augmentation du flux sanguin. Inversement, les neurotransmetteurs du système sympathique, comme la noradrénaline, activent les péricytes (représentés en vert) et induisent une vasoconstriction et donc une diminution du flux sanguin insulaire.

$P C$ insulaires, et à la dilatation d'un sous-groupe de capillaires insulaires, l'augmentation de diamètre étant estimée à $36 \%$. Ces variations de concentration calcique ne sont en revanche pas observées dans les capillaires acinaires ni dans les artérioles pancréatiques.

Les auteurs ont émis l'hypothèse que l'augmentation de l'activité des cellules bêta est associée à une augmentation de la concentration d'adénosine extracellulaire. Ils ont montré dans le modèle CPEV que l'adénosine, ou le $5^{\prime}$-(N-ethylcarboxamido)adenosine (NECA, agoniste non spécifique du récepteur de l'adénosine), reproduisent les effets de l'augmentation du taux de glucose sur les PC insulaires. À l'inverse, la théophylline (antagoniste non spécifique du récepteur de l'adénosine) ou le PSB36 (antagoniste sélectif du récepteur $A l$ de l'adénosine) inhibent l'effet de l'hyperglycémie sur les péricytes insulaires. axones sympathiques sont en contact proche avec les PC exprimant l' $\alpha$ SMA. L'application de 50 à $100 \mu \mathrm{M}$ d'adrénaline, ou de $10 \mu \mathrm{M}$ de noradrénaline, sur les CPEV induit une augmentation importante de la concentration en calcium cytosolique dans les PC et la vasoconstriction d'une partie des capillaires. L'adrénaline a également un effet vasoconstricteur puissant au niveau des artérioles.

Pour tester l'effet de la sécrétion endogène et locale de noradrénaline, Almaça et ses collaborateurs ont appliqué $50 \mu \mathrm{M}$ de tyramine (sympathomimétique qui stimule le relargage des neurotransmetteurs par les terminaisons nerveuses) sur les CPEV : le calcium intracytosolique des $P C$ augmente également.

Dans le modèle de greffe d'îlots en chambre antérieure de l'œil, l'instillation de gouttes ophtalmiques de phényléphrine (un agoniste du récepteur $\alpha$ l de l'adrénaline) provoque une augmentation de la concentration du calcium cytosolique des PC insulaires, une contraction d'une partie des capillaires insulaires, mais aussi une diminution du flux sanguin d'un grand nombre de capillaires.

Les neurotransmetteurs du système sympathique activent donc les PC in vivo, induisent une vasoconstriction et diminuent le flux sanguin insulaire. II est à rapprocher de ceci le fait que la sécrétion d'insuline est inhibée au cours de l'effort physique par des mécanismes dont on sait qu'ils dépendent des catécholamines (Figure 1).

Ce travail est fondamental car il montre comment s'établit le dialogue entre les cellules bêta et leurs capillaires, comment l'augmentation d'activité des cellules bêta conduit à une vasodilatation par action sur les péricytes insulaires et comment des effets systémiques, communiqués par le système nerveux autonome, peuvent moduler la fonction des cellules bêta par réduction du flux sanguin insulaire.

Enfin l'amorce d'observation d'une réduction des péricytes chez des 
patients ayant un diabète de type 2 soulève des interrogations sur le possible rôle d'une dysfonction vasculaire dans la progression des anomalies de sécrétion de l'insuline au cours du diabète de type 2 .

Ce travail pourrait ainsi aller dans le sens d'une intégration des signaux nerveux par le pancréas, mais également d'un rôle important de la microvascularisation dans l'histoire naturelle du diabète de type 2 . $\diamond$

The pericyte of the pancreatic islet regulates capillary diameter and local blood flow

\section{LIENS D'INTÉRÊT}

Les auteurs déclarent n'avoir aucun lien d'intérêt concernant les données publiées dans cet article.

\section{RéFÉRENCES}

1. Logue J, Walker JJ, Colhoun HM, et al. Do men develop type 2 diabetes at lower body mass indices than women? Diabetologia $2011 ; 54$ : 3003-6.

2. Lammert $\varepsilon$. The vascular trigger of type II diabetes mellitus. Exp Clin Endocrinol Diabetes 2008; 116 (suppl 1) : S21-5.

3. Larger $\varepsilon$. Hyperclycémie et angiogenèse. Med Sci (Paris) $2003 ; 19: 840-6$

4. Almaça J, Weitz J, Rodriguez-Diaz R, et al. The pericyte of the pancreatic islet regulates capillary diameter and local blood flow. Cell Metab $2018 ; 27: 630-44 . e 4$

5. Armulik A, Abramsson A, Betsholtz C. Endothelial/ pericyte interactions. Circ Res $2005 ; 97: 512-23$.
6. Hall CN, Reynell C, Gesslein B, et al. Capillary pericytes regulate cerebral blood flow in health and disease. Nature 2014 ; $508:$ 55-60.

7. Peppiatt CM, Howarth C, Mobbs P, et al. Bidirectional control of CNS capillary diameter by pericytes. Nature $2006 ; 443: 700-4$.

8. Sasson A, Rachi $\varepsilon$, Sakhneny L, et al. Islet pericytes are required for $\beta$-cell maturity. Diabetes $2016 ; 65$ : 3008-14.

9. Marciniak A, Cohrs CM, Tsata V, et al. Using pancreas tissue slices for in situ studies of islet of Langerhans and acinar cell biology. Nat Protoc 2014 ; 9 : 2809-22

10. Speier S, Nyqvist D, Cabrera 0 , et al. Noninvasive in vivo imaging of pancreatic islet cell biology. Nat Med $2008 ; 14: 574-8$.

11. Rodriguez-Diaz R, Abdulreda MH, Formoso AL, et al. Innervation patterns of autonomic axons in the human endocrine pancreas. Cell Metabolism 2011 ; $14: 45-54$.

\section{NOUVELle}

\section{Un nouvel éclairage sur l'excitabilité thalamocorticale dans I'épilepsie-absence}

> Jusqu'à récemment, le décryptage du rôle joué par les différentes régions cérébrales dans la génération des rythmes de l'électroencéphalogramme (દદG) reposait sur l'enregistrement d'activités neuronales effectué sur des préparations anesthésiées. De telles préparations sont cependant peu adaptées pour étudier l'activité et la synchronisation des réseaux neuronaux pendant le cycle veille/sommeil ou lors de modifications physio(patho)logiques du niveau de vigilance. Les avancées des techniques électrophysiologiques ont changé la donne. Il est en effet devenu possible au moyen de tétrodes ou d'électrodes multicanaux d'enregistrer simultanément les activités unitaires d'ensembles neuronaux chez des animaux vigiles. Ces enregistrements peuvent être éventuellement combinés à des manipulations de l'activité neuronale permettant d'étudier la contribu- tion de canaux voltage-dépendants et de récepteurs des neurotransmetteurs à la dynamique des réseaux [1].

Lors du sommeil à onde lente ${ }^{1}$, les relations réciproques entre le cortex et le thalamus, constituant la boucle thalamocorticale, sont essentielles à l'émergence d'un grand nombre d'activités rythmiques, particulièrement les fuseaux, les oscillations lentes et de type delta ${ }^{2}$. Dans cette boucle, les neurones glutamatergiques (excitateurs) corticothalamiques et thalamocorticaux sont réciproquement connectés; les neurones corticothalamiques projettent également sur les neurones GABAergiques (inhibiteurs)

\footnotetext{
${ }^{1}$ Le sommeil à ondes lentes est défini négativement comme non-REM sleep, sommeil qui n'est pas le sommeil paradoxal. Un seul critère le définit, la présence de fuseaux et d'ondes lentes cérébrales.

2 Les plus lentes des ondes cérébrales. Elles ont une fréquence faible $(0,1-4 \mathrm{~Hz})$ mais une amplitude forte.
}

${ }^{1}$ Sorbonne Université, CNRS, Inserm, Neuroscience Paris Seine - Institut de Biologie Paris Seine (NPS - IBPS), 9, quai St Bernard, 75252 Paris Cedex 05, France. ${ }^{2}$ Équipe Waking, Centre de recherche en neurosciences de Lyon, CRNL, Inserm U1028, CNRS UMR5292, Université Lyon 1, Lyon, France. nathalie.leresche@upmc.fr

localisés dans le noyau réticulé thalamique (NRT), induisant une inhibition de type feed-forward ${ }^{3}$ des neurones thalamocorticaux. Enfin, les neurones thalamocorticaux projettent en retour sur les neurones du NRT (Figure IA). L'interaction dynamique des différentes structures de la boucle thalamocorticale est également à la base d'activités rythmiques pathologiques, telles celles de l'épilepsie de type absence chez l'enfant. Cette épilepsie non-convulsive est caractérisée par la répétition fréquente de brefs épisodes de perte de conscience associés à l'apparition sur l'દદG d'une oscillation spécifique, les décharges pointe-ondes [2].

L'activité de la boucle thalamocorticale est fortement conditionnée par les propriétés intrinsèques des neurones

\footnotetext{
${ }^{3}$ Effet inhibiteur d'une entrée excitatrice par l'intermédiair d'un interneurone GABAergique intercalé dans le réseau.
} 